\title{
Interpretation of current IceCube results on Galactic and Extragalactic source searches
}

\author{
J. A. Aguilar* for the IceCube Collaboration \\ University of Geneva \\ E-mail: juan.aguilar@unige.ch
}

\begin{abstract}
The IceCube Neutrino Observatory is an array of 5,160 photomultipliers (PMTs) deployed on 86 strings at 1.5-2.5 km depth within the ice at the South Pole. The main goal of the IceCube experiment is the detection of an astrophysical neutrino signal. Three years of IceCube data have been analyzed for the search of neutrino point-sources. In the case of point-sources of neutrino emission, the IceCube neutrino telescope is sensitive to sources with $\mathrm{E}^{-2}$ spectra mainly in the TeV-PeV energy range in the northern sky. In the opposite hemisphere the detector is most sensitive to sources with harder spectra to which IceCube is mostly sensitive between PeV-EeV energies. In the absence of evidence for a cosmic signal from a point-source in these there years of data the upper limits in the neutrino flux are set. These limits are constraining the parameter space of models of neutrino emission from both galactic and extra-galactic sources as shown in this contribution.
\end{abstract}

XV International Workshop on Neutrino Telescopes

March 11-15 2013

Venice, Italy

\footnotetext{
${ }^{*}$ Speaker.
} 


\section{Introduction}

The main scientific goal of the IceCube experiment is the detection of astrophysical neutrinos that will help to understand and settle the unresolved questions about the origin and nature of Cosmic Rays (CRs). The random-walk of CR particles through the intergalactic magnetic fields makes it impossible to directly identify the cosmological sources of CRs. On the other hand, neutrinos are likely produced in the same environmental condition as CRs and $\gamma$-rays and, being electrically neutral, they propagate directly from the source to the Earth. In addition, neutrinos only interact weakly and can therefore pass dense regions of the Universe without being attenuated. The detection of high-energy cosmic neutrinos will provide a direct proof of hadronic particle acceleration in the Universe since they can only be produced by the interactions of protons or nuclei with ambient radiation or matter. Since these astrophysical neutrinos also carry directional information, their detection will make it possible to unequivocally identify the sources of CRs and connect their acceleration to Supernova Remnant (SNR) shocks, Active Galactic Nuclei (AGN) jets or Gamma-Ray Bursts (GRBs) [1].

In these proceedings we present the results of the point source data analysis from 2008 to 2011 when three different detector configurations were operated. The description of the different geometries and the corresponding event selection is given in Sec. 2. The results of the IceCube data analysis corresponding to three years of data are given in Sec. 3 and its implication on some models of astrophysical neutrino emission in Sec. 4. Conclusions are given in Sec. 5.

\section{Detector and analysis}

The IceCube Neutrino Observatory was designed to detect neutrinos of an astrophysical origin with its in-ice Cherenkov detector and Cosmic Rays at the energies around the knee $\left(\sim 3 \times 10^{15}\right.$ $\mathrm{eV}$ ) with a surface array detector (IceTop). The in-ice detector uses a cubic kilometer volume of clear Antarctic ice underneath the geographic South Pole. This volume is instrumented with an array of 5,160 Digital Optical Modules (DOMs) deployed on 86 strings from 1.5-2.5 km deep [2]. The DOMs are spherical, pressure resistant glass housings containing each a $25 \mathrm{~cm}$ diameter Hamamatsu photomultiplier tube (PMTs) and electronics for waveform digitalization. The in-ice IceCube detection principle is based on the measurement of the Cherenkov light induced by ultra-relativistic leptons produced by neutrino interactions in the matter surrounding the detector.

The detector was completed in December 2010. Previously various incomplete configurations were taking data. Fig. 1 shows the string layout of the 40-string configuration (IC-40) which took data from 2008 April 5 to 2009 May 20, the 59-string configuration (IC-59) active from 2009 May 20 to 2010 May 31, and the 79-string configuration (IC-79) active from 2010 May 31 to 2011 May 13.

At trigger level the majority of the recorded events from the IceCube detector are muons produced by $\mathrm{CR}$ interactions in the atmosphere. These atmospheric muons constitute the largest contribution of background events in IceCube. A first level of background rejection is done on-site at the South Pole reducing the trigger rate by about an order of magnitude. A satellite link is used to transmit the data that pass the initial filter to the North where further processing is performed including a broader range of more CPU consuming likelihood-based reconstructions. The final 


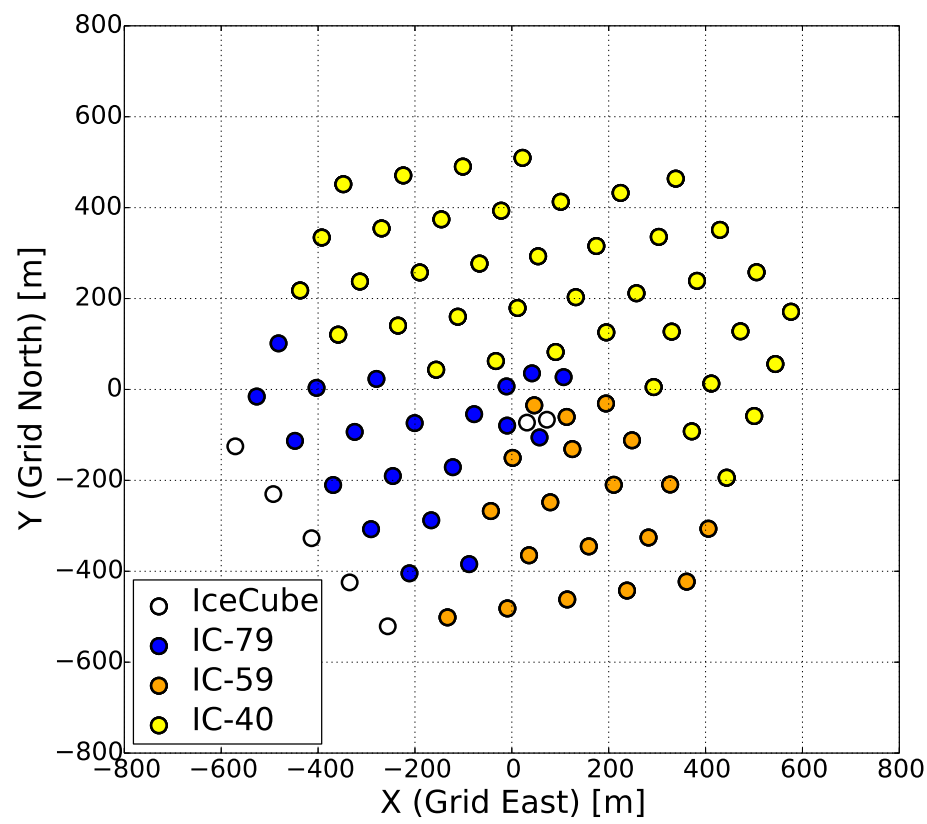

Figure 1: Detector layout of IceCube. The circles represent the surface string positions corresponding to the final geometry of the whole IceCube detector. The IC-40 configuration is represented by yellow dots. The green circles represent the additional strings that form the IC-59 configuration. The IC-59 configuration together with the strings indicated by blue circles represent the IC-79 configuration.

Table 1: Summary of the three different IceCube configurations used in this analysis. We show the expected atmospheric neutrino rate from MC simulation weighted by the atmospheric neutrino flux calculation in Ref. [4] and the numbers of up- and down-going events at final selection level.

\begin{tabular}{ccccc}
\hline configuration & live-time [days] & atm. $v$ s & \# up-going & \# down-going \\
\hline IC-40 & 376 & 40/day & 14,121 & 22,779 \\
IC-59 & 348 & 120/day & 43,339 & 64,230 \\
IC-79 & 316 & 180/day & 50,857 & 59,009 \\
\hline
\end{tabular}

event selection lowers the background to the level of atmospheric neutrinos in the northern sky and to a reduced sample of atmospheric muons in the southern sky. This event selection is done using a combination o simple cuts on observables with discrimination power between signal (generally an $\mathrm{E}^{-2}$ neutrino signal) and background (atmospheric neutrinos and muons) but also using more sophisticated multi-variate classification techniques like Boosted Decision Tree (BDT) [3]. Table 1 summarizes the live-time, the estimated rate of atmospheric neutrinos and the number of up-going and down-going track events in the three different configurations. 

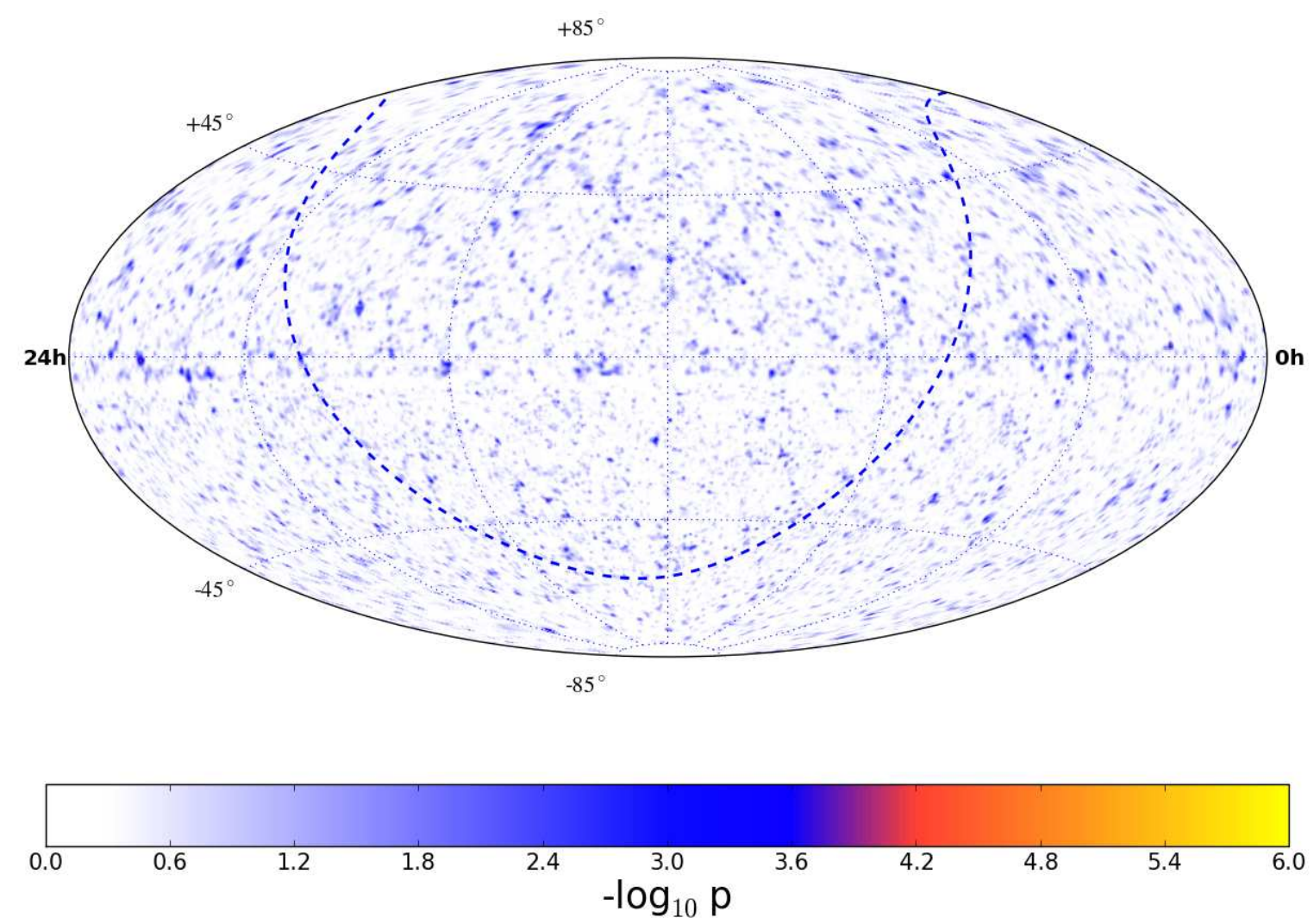

Figure 2: Pre-trial significance skymap in equatorial coordinates (J2000) of the all-sky point source scan for the combined IC79+IC59+IC40 data sample. The dashed line indicates the galactic plane.

\section{Time-integrated searches of neutrinos.}

IceCube uses a likelihood method for the search of neutrino point-sources. This method models the expected signal from a point source in the sky using neutrino simulation and the background estimate using real data. The maximum likelihood ratio makes it possible to calculate the significance of an excess of neutrinos above the background for a given direction.

In time-integrated searches the signal hypothesis is a steady point (or slightly extended) source of neutrinos. In particular the results of the whole sky scan of point-sources of neutrinos are shown in Fig. 2. This figure shows the pre-trial significance map where the most significant deviation in the northern sky has a pre-trial $p$-value of $1.96 \times 10^{-5}$ and is located at $34.25^{\circ}$ r.a. and $2.75^{\circ}$ dec. Similarly, the most significant deviation in the southern sky has a pre-trial $p$-value of $8.97 \times 10^{-5}$ and is located at $219.25^{\circ}$ r.a. and $-38.75^{\circ}$ dec. The post-trial probabilities calculated as the fraction of similar experiments (scrambled sky maps) with at least one spot with an equal or higher significance for each hemisphere correspond to $57 \%$ and $98 \%$ for the northern and the southern spots respectively.

Since no significant excess was found in this analysis we set upper limits on the neutrino flux 


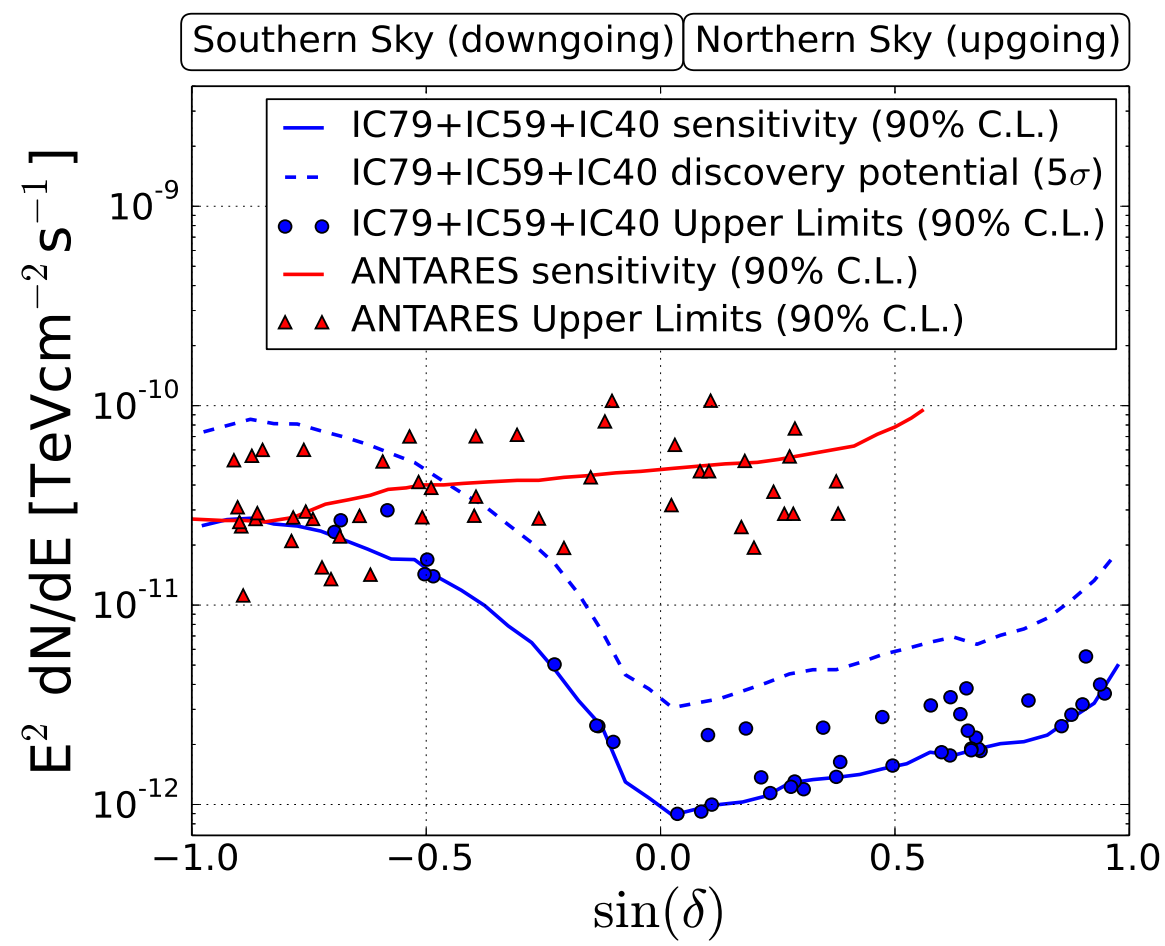

Figure 3: Muon neutrino and antineutrino flux $90 \%$ C.L. upper limits for an $\mathrm{E}^{-2}$ spectrum. Published limits of ANTARES [17] are also shown.

coming from some potential sources. Figure 3 shows the IceCube sensitivity defined as the median upper limit and the $90 \%$ C.L. upper limits for a list of individual sources. The upper limits in the muon neutrino flux shown in Fig. 3 already prove that IceCube sensitivity is comparable to the observed $\gamma$-ray fluxes [23].

\section{Implication on time-integrated sources}

A non discovery for IceCube has meaningful implications on some astrophysical models of neutrinos emission mechanisms and can provide insight about the nature of these phenomena. In this section we will discuss these implication for models of neutrino emission in the Galaxy and for extra-galactic sources as well by comparing the upper limits from the point-source analysis to the neutrino predictions. The results of the diffuse neutrino emission search and its implication are not considered in the following [22].

\subsection{Galactic sources}

IceCube has provided the most constraining upper limits on neutrino fluxes from galactic sources like the Crab [7]. The Crab spectral emission seems to be fully explained by electromagnetic phenomena [8] however several $\gamma$-ray flares observed in the past years in the $\mathrm{GeV}$ region $\left(E_{\gamma}>100 \mathrm{MeV}\right)$ challenge the purely leptonic models [16]. 


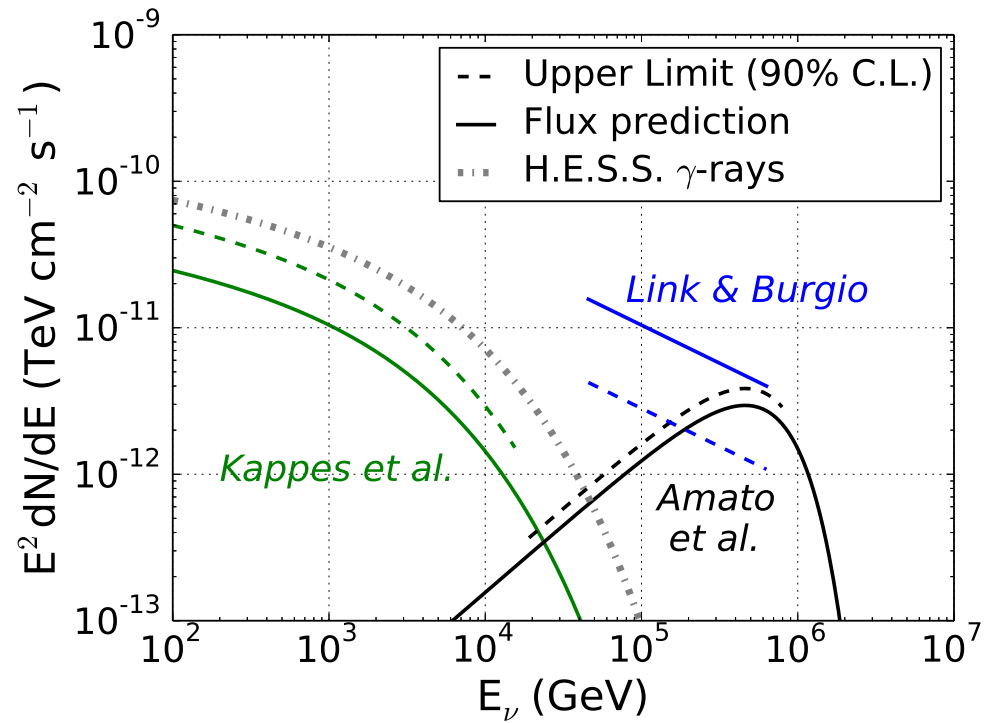

Figure 4: Predicted muon neutrino fluxes for several hadronic models ([9], [13], [14]) for steady neutrino emission from the Crab and upper limits based on 3 years of IceCube data. Solid lines indicates the flux prediction and the dashed lines the corresponding upper limit flux for a 90\% C.L. for an energy range that contains $90 \%$ of the signal.

Figure 4 shows the expected neutrino flux at Earth from the Crab according to three different models together with the corresponding $90 \%$ C.L. neutrino upper limits. As a reference the $\gamma$-ray spectrum measured by H.E.S.S. [10] is also shown.

The green solid line represents the neutrino flux based not the measured $\gamma$-ray according to [9]. As can be seen, the corresponding IceCube limit (dashed line) is between the neutrino prediction and the observed $\gamma$-ray spectrum.

The black line represents the estimated flux based on the resonant cyclotron absorption model [12, 13] for the most optimistic case of the effective target density and with a wind Lorentz factor of $\Gamma=10^{7}$ while the favored values of the upstream Lorentz factor of the wind are $\Gamma=10^{6}[11]$. In this case the upper limit is close to reject this optimistic realization of the model.

The third model is rejected with more than $90 \%$ C.L. corresponding to the most optimistic case described in Ref. $[14,15]$ were scattering of wind protons with the X-ray emission from the pulsar's surface is considered assuming a quadratic scaling of the proton's energy with the height above the surface.

In addition to the Crab, IceCube upper limits are also approaching the predicted neutrino emission from SNRs. In the northern sky, G40.5-0.5 [19] is one the most promising SNR candidates for a neutrino detection due to its high $\gamma$-ray flux. The authors in [18] calculated the neutrino spectra generated by $p p$ interactions in this and other SNRs. Figure 5 shows the predicted muon neutrino spectra for this an other two SNRs, Cas A and IC443, as well as the 90\% C.L. flux upper limits. As can be seen none of these sources can be excluded at more than $90 \%$ C.L. assuming that the $\gamma$-ray flux has a cut-off.

One of the most interesting galactic region for IceCube is the Cygnus region which is the 


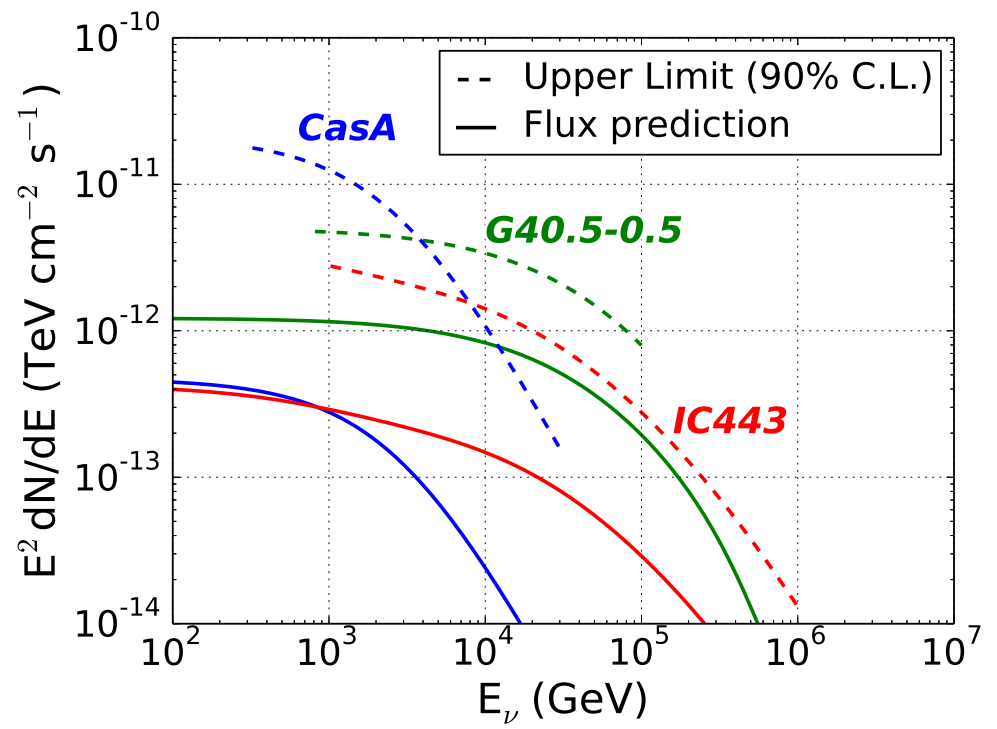

Figure 5: Predicted muon neutrino fluxes and upper limits from three SNRs in the northern sky according to the prediction of Ref. [18].

brightest extended region in the entire northern sky in $\mathrm{TeV} \gamma$-ray [5]. An specific stacking analysis on 6 sources detected by Milagro in the $\mathrm{TeV} \gamma$-ray was performed. Four of these sources are in the Cygnus region. For the 3 years analysis of IceCube the result of this analysis resulted in a post-trial $p$-value of $20.4 \%$.

Figure 6 shows the $90 \%$ C.L. upper limit based on this result for the 6 Milagro TeV $\gamma$-ray associations assuming the model from Ref. [6]. Since the result of the analysis was a positive fluctuation the upper limit is higher than the corresponding sensitivity.

\subsection{Extra-Galactic sources}

High energy CRs are believed to be accelerated by powerful extragalactic sources, like GRBs, AGNs or starburst galaxies. Extra-galactic sources of cosmic rays are more difficult to detect with a point-source analysis and most probably, if the cosmic ray accelerator are numerous and spread over the sky, a diffuse neutrino emission will more likely be detected before the corresponding point-source flux. Nonetheless, the current sensitivity of IceCube can already constrain the parameter space of purely hadronic scenarios of activity of blazars. In [23] the authors made an exploration of hadronic interactions in blazars using only the IceCube sensitivity of the 40-string configuration. When $p p$ interactions dominate some constrains on the primary proton spectrum can be imposed assuming that the resulting neutrino spectrum from $p p$ interaction cannot exceed the IC-40 sensitivity. In particular, for 3C 454.3, the very high energy part of the spectra of blazars is constrained to be harder than $\mathrm{E}^{-2}$ with cut-off energies in the range of $\mathrm{E}_{c u t}>10^{18} \mathrm{eV}$ (see Fig. 7, taken from Ref. [23]).

A similar approach is followed in Ref. [24] where the authors consider $p p$ interaction models in Fanaroff-Riley type I (FR-I) radio galaxies instead of blazars. The authors use the flux limits on the neutrino emission provided by the 40-string configuration of IceCube [25] and the $\gamma$-ray 


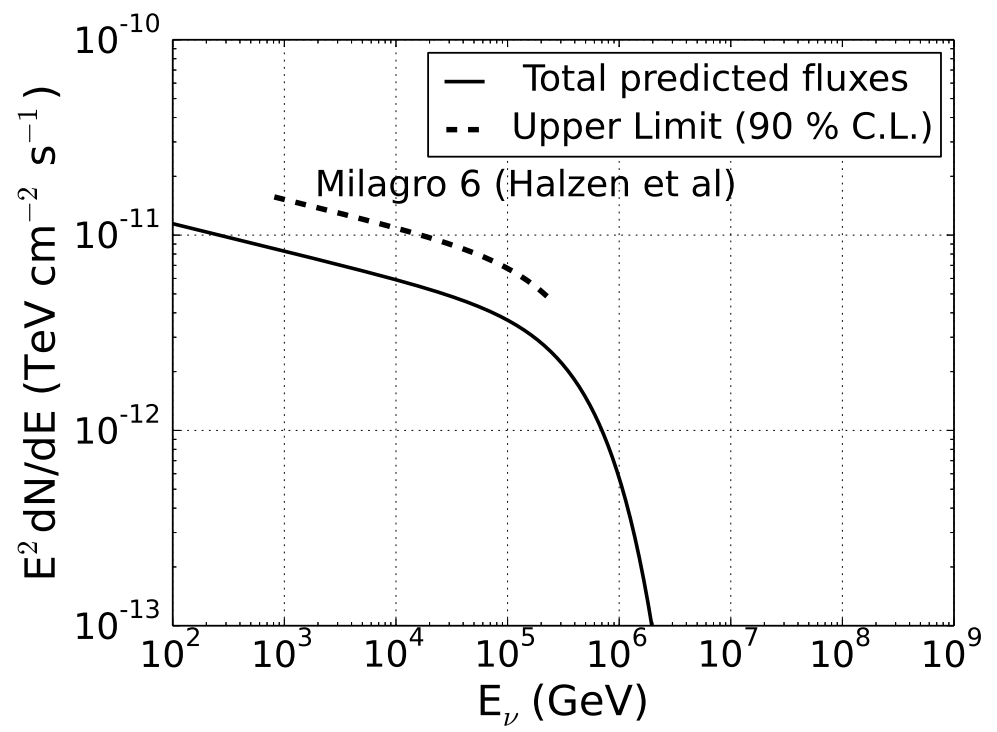

Figure 6: Predicted muon neutrino fluxes from 6 Milagro sources in $\gamma$-rays according to [6]. The corresponding $90 \%$ C.L. flux upper limit for muon neutrinos obtained from the stacking analysis are shown as well.

observations to limit the source properties in particular the target density $n_{H}$ for $p p$ interaction. The result of the study showed that the limit on the target density for 33 FR-I galaxies can be found to be smaller than $30 \mathrm{~cm}^{-3}$ to $2 \cdot 10^{3} \mathrm{~cm}^{-3}$ depending on the source for fixed value of the ratio of electrons to protons of $f_{e}=0.1$. For two particular cases, Centaurus A and Messier 87, they also use Fermi observations to model the $\gamma$-flux, the neutrino flux and the resulting target density. In this case they found that the expected neutrino flux is below the sensitivity of the 40-string configuration of IceCube.

\section{Conclusions}

IceCube is entering a stage in which a non-discovery of a point-source has meaningful and quantitative implication on astrophysical models of cosmic ray and neutrino emission. We showed how the recent results of IceCube concerning the search of steady point-sources of neutrinos already rejects the most optimistic models of neutrino emission at 90\% C.L. for sources like the Crab. For extra-galactic sources some studies showed that in purely hadronic scenarios some constrains can be set using previous limits for only the 40-string configurations.

\section{References}

[1] J. Becker, Phys. Rep. 458 (2007) 173-246.

[2] R. Abbasi et al. [IceCube Coll.], Nucl. Inst. Meth. A 618 (2010) 139.

[3] M. Aartsen et al. [IceCube Coll.], Accepted for publication Astrop. J. (2013).

[4] M. Honda et al., Phys. Rev. D75 (2007) 043006. 


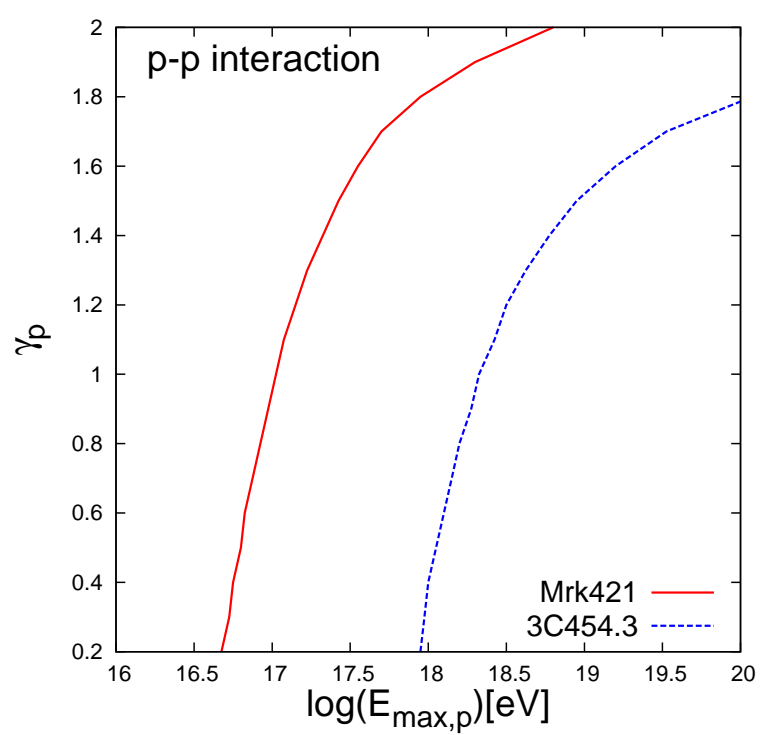

Figure 7: Exclusion plot for $p p$ interactions model parameter. The excluded area is left and above each line. This plot corresponds to the cascade dominated $\gamma$-ray emission case considered in Ref. [23].

[5] A. A. Abdo et al. [Milagro Coll.], Astrop. J. 658 (2007) L33.

[6] F. Halzen, A. Kappes, and A. O’Murchadha, Phys. Rev. D78 (2008) 063004.

[7] R. Abbasi et al. [IceCube Coll.], Astrop. J. 745 (2012) 45.

[8] J. J. Hester, Ann. Rev. Astron. Astrophys. 46 (2008) 127.

[9] A. Kappes et al., Astrop. J. 656 (2007) 870.

[10] F. Aharonian et al. [HESS Coll.], Astron. Astrophys. 457 (2006) 899.

[11] Y. A. Gallant \& J. Arons, Astrop. J. 435 (1994) 230.

[12] W. Bednarek, Astron. Astrophys. 407 (2003) 1.

[13] E. Amato, D. Guetta, and P. Blasi, Astron. Astrophys. 402 (2003) 827.

[14] B. Link \& F. Burgio, Phys. Rev. Lett. 94 (2005) 181101.

[15] B. Link \& F. Burgio, Mon. Not. Roy. Astron. Soc. 371 (2006) 375.

[16] A. A. Abdo et al. [Fermi Coll.], Science 331 (2011) 739.

[17] S. Adrián-Martínez et al. [ANTARES Coll.], Astrop. J. Lett. 743 (2011) L14.

[18] M. Mandelartz \& J. Becker Tjus, subm. to Astrop. J. (2013) [arXiv:1301.2437].

[19] N. E. Kassim, Astrophys. J. Suppl. Ser. 71 (1989) 799.

[20] J. Albert et al. [MAGIC Coll.], Astrop. J. 664 (2007) L87.

[21] A. A. Abdo et al. [Milagro Coll.], Astrop. J. 712 (2010) 459.

[22] M. G. Aartsen et al. [IceCube Coll.], Science 342, 1242856 (2013).

[23] C. Tchernin et al., Accepted for publication Astron. Astrophys. (2013) [arXiv:1305.3524].

[24] I. Saba, J. Becker Tjus, and F. Halzen, Astropart. Phys. 48 (2013) 30-36.

[25] R. Abbasi et al. [IceCube Coll.], Astrop. J. 732 (2011) 18. 